\section{Stent als Erektionshelfer}

Vaskuläre Probleme gehören zu den wichtigsten Ursachen einer organisch bedingten erektilen Dysfunktion. Die Einführung von PhosphodiesteraseInhibitoren hat eine Verbesserung für viele Patienten ergeben, jedoch sprechen bis zu 50\% der Patienten nur suboptimal darauf an. Profitieren diese Patienten von einer endovaskulären Therapie?

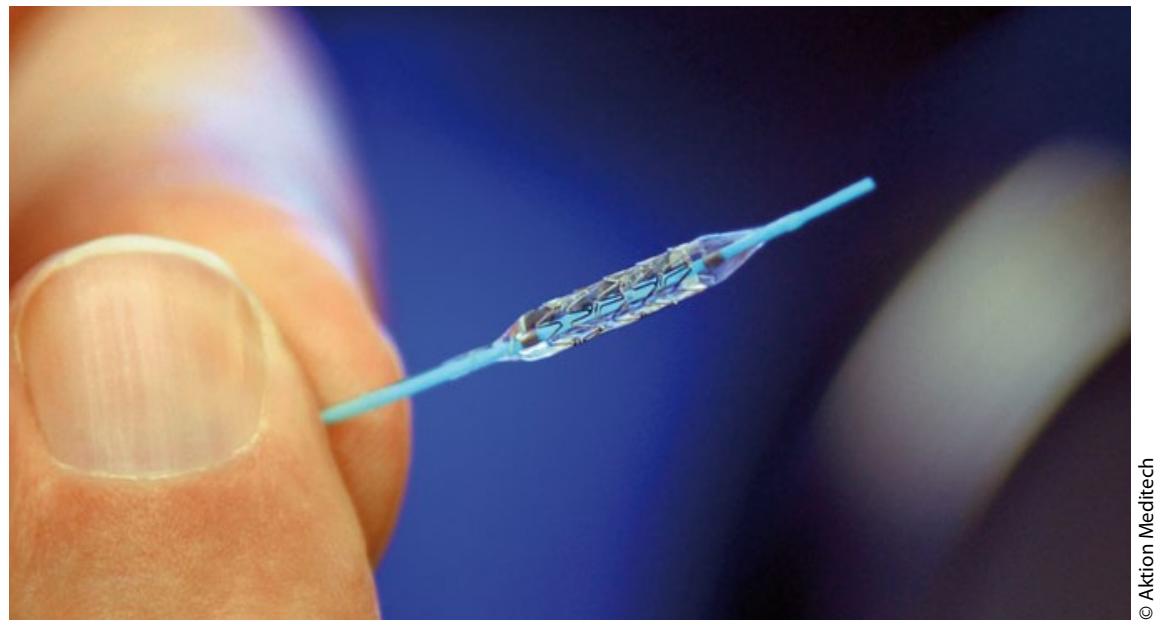

Koronarstents können auch Penisarterien offen halten.

$B$ ei 30 Männern mit einer angiografisch verifzierten Stenose einer oder beider Aa. pudendae internae wurden medikamentenbeschichtete Koronarstents implantiert. Die Männer waren etwa 60 Jahre alt und hatten auf Phosphodiesterase-Inhibitoren suboptimal angesprochen. Alle Patienten waren zuvor hinsichtlich einer penilen Minderperfusion mithilfe der Duplexsonografie der A. pro- funda penis nach intrakavernöser Injektion eines Prostanoids gescreent worden. Der technische Erfolg der Katheterintervention, bei der Arterien mit einem mittleren Durchmesser von 2,6 mm behandelt worden waren, betrug $100 \%$. Nach der Stentimplantation hatten knapp $60 \%$ der dilatierten Patienten eine funktionelle Verbesserung bei verbesserter peniler Durchblutung. Nach sechs Monaten be- trug die angiografisch verifizierte Restenoserate $34 \%$.

\section{Kommentar}

Die Revaskularisierung bei erektiler Dysfunktion könnte aufgrund der vorliegenden Daten aufleben. Technische Verbesserungen und Miniaturisierungen lassen heute eine endovaskuläre Therapie nicht nur der Beckenstammgefäße, sondern auch der den Penis versorgenden Arterien des kleinen Beckens zu. Neben den funktionellen Auswirkungen auf die Erektion, die für die betroffenen Patienten eine deutliche Besserung der Lebensqualität nach sich ziehen können, kann die Entdeckung einer vaskulär bedingten erektilen Dysfunktion bei bisher ansonsten kardiovaskulär blanden Patienten ein wichtiger Frühindikator für eine sich manifestierende Atherosklerose sein. Weitere Studien auf diesem klinisch bisher vielerorts vernachlässigten Gebiet sollten den Stellenwert der endovaskulären Therapie gegenüber rein konservativen Therapiemaßnahmen untersuchen. Angesichts der vorliegenden Zahlen aus der ZEN-Studie scheint eine angiologische Untersuchung von Patienten mit erektiler Dysfunktion, bei denen kardiovaskuläre Risikofaktoren bekannt sind, durchaus sinnvoll.

Prof. Nicolas Diehm

Rogers JH et al. Zotarolimus-eluting peripheral stents for the treatment of erectile dysfunction in subjects with suboptimal response to phosphodiesterase-5 inhibitors. J Am Coll Cardiol 2012; 60: 2618-27 